\title{
Damage-based long-term modelling of a large alpine rock slope
}

\author{
Federico Riva (1), Federico Agliardi (1), David Amitrano (2), and Giovanni B. Crosta (1) \\ (1) Department of Earth and Environmental Sciences, Università degli Studi di Milano - Bicocca, Milano, Italy, (2) ISTerre, \\ IRD, CNRS, OSUG, Universite’' de Grenoble, Grenoble, France.
}

The morphology and stability of large alpine rock slopes result from the long-term interplay of different factors, following a complex history spanning several glacial cycles over thousands of years in changing morpho-climatic settings. Large rock slopes often experience slow long-term, creep-like movements interpreted as the macroscopic evidence of progressive failure in subcritically stressed rock masses. Slope damage and rock mass weakening associated to deglaciation are considered major triggers of these processes in alpine environments. Depending on rock mass properties, slope topography and removed ice thickness, valley flanks can progressively evolve over time into rockslides showing seasonal displacement trends, interpreted as evidence of hydro-mechanically coupled responses to hydrologic perturbations.

The processes linking the long-term evolution of deglaciated rock slopes and their changing sensitivity to hydrologic triggers until rockslide failure, with significant implications in risk management and Early Warning, are not fully understood. We suggest that modelling long-term rock mass damage under changing conditions may provide such a link.

We simulated the evolution of the Spriana rock slope (Italian Central Alps). This is affected by a $50 \mathrm{Mm} 3$ rockslide, significantly active since the late 19th century and characterized by massive geological and geotechnical investigations and monitoring during the last decades. Using an improved version of the 2D Finite-Element, damage-based brittle creep model proposed by Amitrano and Helmstetter (2006) and Lacroix and Amitrano (2013), we combined damage and time-to-failure laws to reproduce diffused damage, strain localization and the long-term creep deformation of the slope. The model was implemented for application to real slopes, by accounting for: 1) fractured rock mass properties upscaling based on site characterization data; 2) fluid pressures in a progressive failure context, relating fluid occurrence to damage.

Starting from a geological and topographic setting consistent with the end of the Last Glacial Maximum, we simulated ice removal in terms of transient surface loading conditions. Results show that deglaciation exerted a major control on the spatial and temporal patterns of slope weakening and subsequent destabilization via progressive rock mass damage and related fluid pressure onset in the slope. The model was validated by comparison to field and borehole data and the spatial and temporal patterns of long-term slope instability were discussed.

Results show the ability of our model to correctly predict in one modelling framework (1) the rockslide geometry and the staged morphological evolution related to creep regime, (2) the macroscopic spatial distribution of damage with consequences on fluid pressure distribution and (3) the long-term evolution of rock mass mechanical properties. 